\title{
The relation between category and magnitude estimates of tactile intensity*
}

\author{
ROBERT H. GIBSON† and DAVID L. TOMKO \\ Departments of Paychology and Pharmacology \\ University of Pittsburgh, Pittsburgh, $\mathrm{Pa} .15213$
}

\begin{abstract}
Magnitude estimates of a wide intensive range of electrotactile stimuli were lotted against two category scales of the same stimuli. One scale was obtained rith the usual narrow range of categories, the other with an expanded range lerived from the numerical magnitude estimates. With the narrow range of ategories, the function relating category ratings to magnitude estimates was urved concave downward as is usual. With the wide range of categories, the unction was linear. Thus, the curvature previously ascribed to prothetic ontinua in general was found rather to result from a specific property of the esponse dimensions, i.e., from the restricted set of numbers made available as ategories to the Ss.
\end{abstract}

Magnitude eatimates of the apparent ntensity of stimuli grow roughly as a ower function of physical intensity or many prothetic sensory continua e.g., vibration (Gibson, 1960), lectrotactile intencity (Gibson, Dillmann, \& Tomko, 1967), brightnees ind loudnews (Stevens, J. C., \& Hall, 1966), and electric shock intensity Stevens, S. S., Carton, \& Shickman, [958)]. On such continua, category atino are related to magnitude timates of the came stimuli by a function which is ordinarily concave lownward (Btevens, S. S., \& Galanter, (957). Because this curved relation colds primarily for prothetic, and not usually for metathetic, continua such 3s the pitch of high frequencies, it has been sugrested a a criterion for identifying prothetic continua (Stevens, S. S., \& Galanter, 1957).

However, the curved nature of this function is perplexing, as it implies that there is not a simple correspondence between magnitude estimates and category ratings on the same intenrive dimension. If category rating and magnitude extimates both measure the same dimension of apparent intemity, the function relating them might be expected to be linear.

These two sealing procedures differ in one respect which may be relevant to this problem. In marnitude eatimation, 0 eolects and uses a range of numbers which represents his range

\footnotetext{
*The research was supported in pert by Grants NB 06304 from the National Grants NB 0630 from the National Stroke and HR8K I-II from the Health Research and services Foundation to the int author. The revench was earried out while the eccond euthor was a prodoctoral NIF trainee and was supported by Peychobioloty Trainine Grant MF 11402 to the Departments of Paycholosy and Pharmacolory, Untveraity of Pittuburth.

+ Now Viatins Reweareh Profewor, Xaviex College, Sydney, N.S., Canada. Send reprint requent to R. H. Gibson, Box 1327 , sydney, N.S., Canuda.
}

of apparent intensities. By contrast, in category rating, $\mathrm{E}$ chooses an arbitrary range of categories for use by $O$. This range, frequently eight or so categories, is ordinarily smaller, though sometimes larger, than the range which is chosen naturally by $O$ in magnitude estimation. If the natural expreation of numerical relationships between the apparent intensities of a number of stimuli depends on the set (range and/or number) of categories aviliable, the typical category rating procedure may constrain $O^{\prime}$ judgments. Thus, the category ratings may not repreaent the growth of apparent intenrity in the same way as do magnitude estimates, with the result displayed as a nonlinear relationship between the two.

The purpose of the present experiment was to determine the effect of the range of categories available to $O$ on the function relating category and magnitude extimates of the stimuli. The apparent intensity of a wide range of tactile electric stimuli (cf. Gibson, 1968) was sealed, using two sets of categories. One set of seven categories comprised the cardinal numbers 2-8, the use of which was expected to provide the usual curved function. The second set of 49 categories comprised the cardinal numbers 2-50 and was derived from the mean range of numbers reported as magnitude estimates of the same stimuli. The essential point of the experiment was to determine the shape of the function relating these category ratings with the magnitude estimates.

\section{METHOD}

Observers

Thirteen male and 20 female University of Pitsburgh undergraduates served as $\mathrm{Os}$.

\section{Stimuli}

Eighty-millisecond-long trains of 0.5-msec anodal rectangular pulses were presented at a repetition rate of 100 pps. Thus, each $80-\mathrm{msec}$ stimulus consisted of nine brief pulses, identical in duration, with 10-msec interpulee onset intervals. Only pulse amplitude was manipulated to vary stimulus intensity. At moderate intensities, such a train of pulses feels like a single brief tap (Gibson, 1968).

\section{Apparatu}

Stimuli were delivered to the palmar base of the right thumb (thenar eminence) through a flat 16-mm-diam stainles steel dirk electrode embedded flush in a 38-mm-diam Plexigls surround disk. The function of the surround was to separate the mechanical and electrical edges of the stimulator, thereby ensuring no pain arousal. The large indifferent aluminum electrode covered the sole of the right foot.

The Tektronix 160 series pulse and waveform generator in Fig. 1 initiated and shaped the pulve train. Train
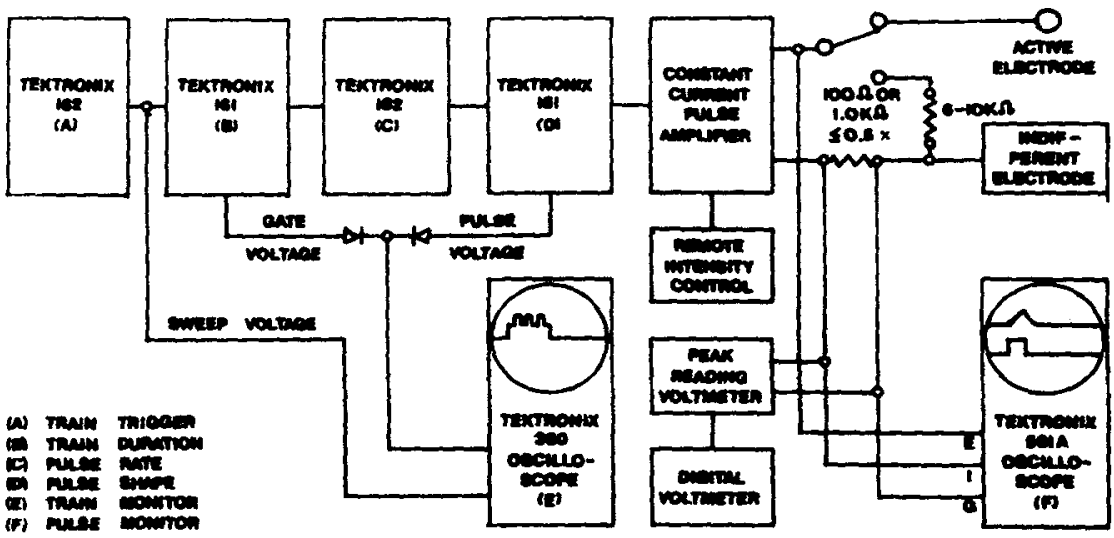

Fig. 1. Stimulus generntion and monitoring equipment. 


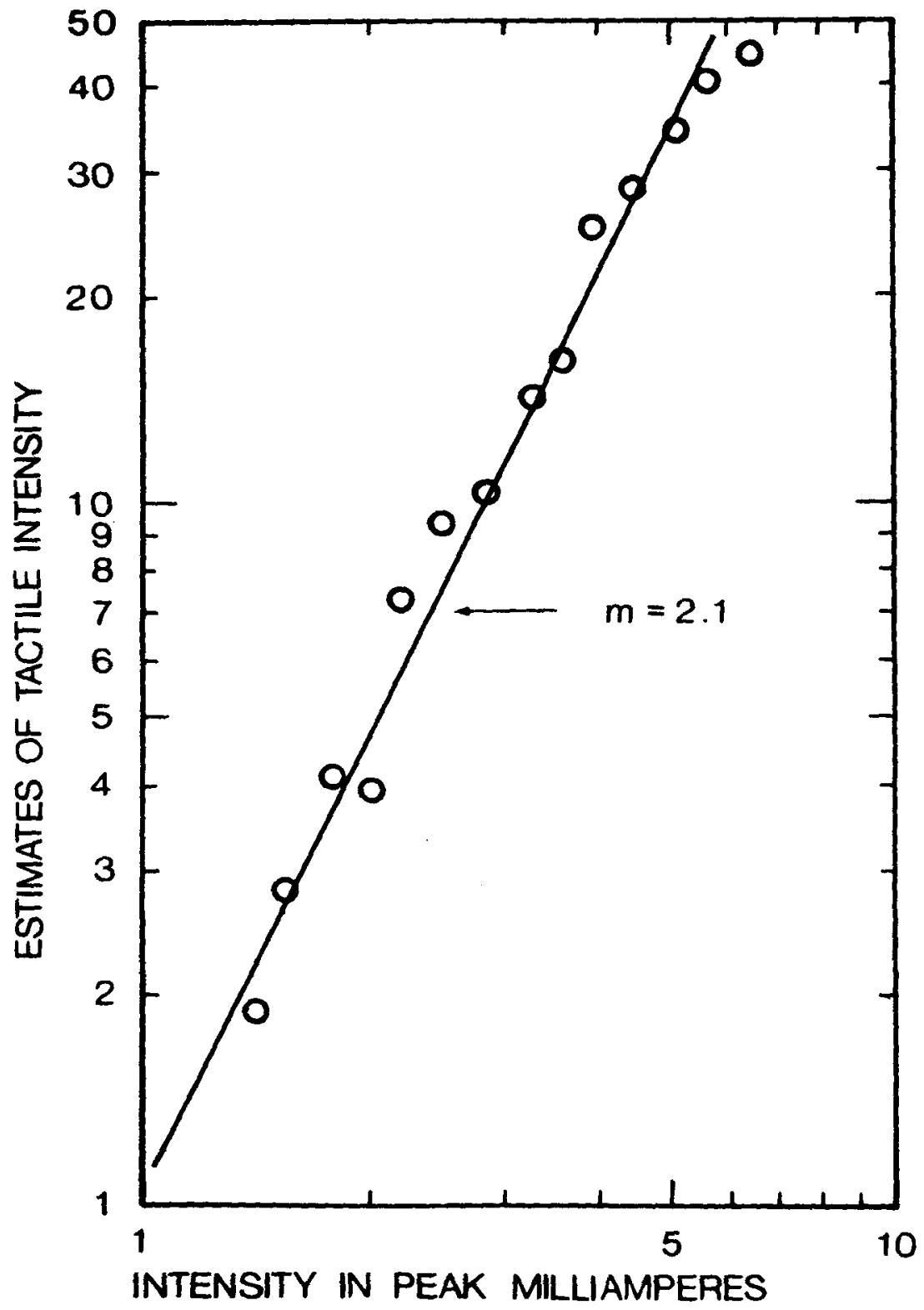

Fig. 2. Geometric mean magnitude estimates plotted as a function of stimulus intensity in peak milliamperes.

duration was monitored by using a Tektronix 360 oscilloscope with input shown in the block diagram. The pulse trains were fed into a constant-current pulse amplifier which supplied a pulse with amplitude continuously variable from 0.1 to $10.0 \mathrm{~mA}$.

During stimulation, $O^{\prime}$ s interelectrode tissue resistance (between the active and indifferent electrodes) was placed across the amplifier output, in electrical series with a 100-ohm precision measuring resistor (shown in Fig. 1 as a 100-ohm or a $1.0 \mathrm{~K}$ ohm resistor). For calibration, a $6.8 \mathrm{~K} \mathrm{ohm} \mathrm{resistor}$ (shown in Fig. 1 as a $6.0 \mathrm{~K}$ ohm-10.0 $\mathrm{K}$ ohm resistor) replaced 0 . the voltage across the precision resistor. Peak current was displayed as milliamperes on the digital voltmeter shown in the block diagram. The pulse shape was monitored by a Tektronix 561A oscilloscope (Fig. 1) in electrical parallel with the measuring resistor.

\section{Procedure}

Each $O$ served in a single 50-min session of two parts. The first part of the session was identical for each $O$ and consisted of nine ascending method of limits series threshold measurements. In the second part of each session, $O$ scaled the apparent intensity of suprathreshold stimuli using one of three scaling procedures: (1) magnitude estimation, (2) category rating using the whole numbers $2-8$ as categories, or (3) category rating with the range of categories derived from the mean range of magnitude estimates.

The stimuli covered the range from 1 to $14 \mathrm{~dB}$ above each $O$ 's threshold, in 1-dB steps. The most intense stimulus, about five times threshold, was reported to feel like a very strong touch. Thus, the perceptual range was quite wide, from barely suprathreshold to nearly intolerably strong.

\section{Magnitude Estimation}

Fourteen stimuli were presented in a different random order to each of 11 Os. They covered the range from 1 to $14 \mathrm{~dB}$ above threshold in 1-dB steps. A modulus-free method of magnitude estimation was used by $O$ to rate the apparent intensity of the stimuli. He was instructed to assign a number to each stimulus which was proportional to its strength. The 4-dB stimulus was always presented for judgment first. It was then repeated for judgment as the middle (seventh) stimulus in the otherwise random array of 14 stimuli.

\section{Category Rating}

The same 14 stimuli were presented to 11 other $O s$ with the same random orders as above. The category rating procedure used was as follows: $O$ was instructed to assign each stimulus to a category representing its strength, using as categories the whole numbers 2-8. The first standard stimulus presented to each $O$, an example of Category 2, was $1 \mathrm{~dB}$ above threshold. The second standard stimulus, presented as Category 8, was $14 \mathrm{~dB}$ above threshold. In an effort to have categories based on equal intensive intervals, $\mathbf{O}$ was further instructed to assign the stimulus halfway between the highest and lowest intensities to the category midway between the standards. In order to eliminate end effects, $O$ was told to use Categories 1 and 9 to represent intensities exceeding the standards. Each of the 12 remaining stimuli ( 2 to $13 \mathrm{~dB}$ above threshold in 1-dB steps) was then presented once each for rating.

\section{Categories Derived from \\ Magnitude Estimates}

The procedure for category rating stimuli (by 11 different $O_{8}$ ) was the same, with these exceptions: The categories were the whole numbers range assigned by the $O s$ during magnitude estimation. The first stimulus (Category 2) was $1 \mathrm{~dB}$ above threshold; the second was $14 \mathrm{~dB}$ (Category 50). O was told to use Categories 1 and 51 to represent $2-50$, whose range approximated the 


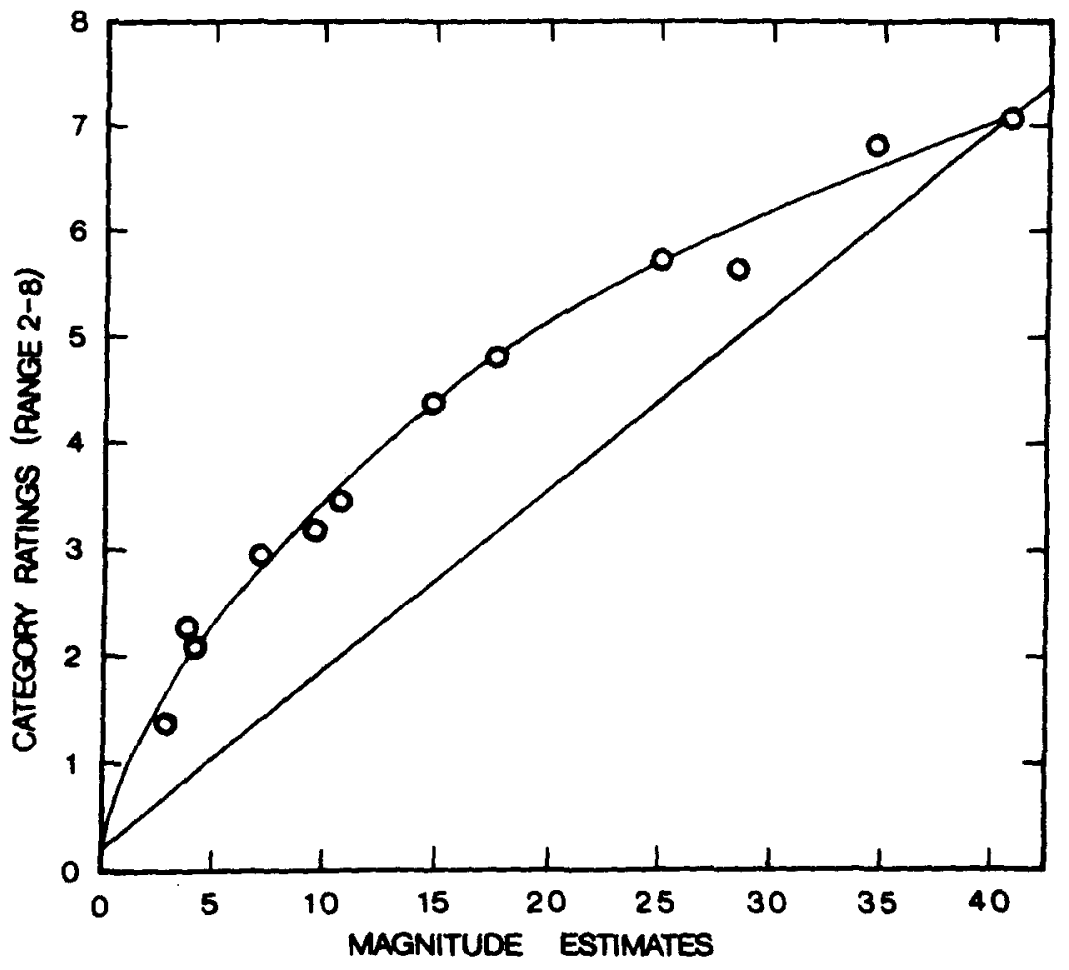

Fis. 3. Arithmetic mean category ratings plotted as a function of geometric mean magnitude extimates of electrotactile timuli between 2 and $13 \mathrm{~dB}$ above threahold. Categories were the whole numbers $2-8$.
In Fig. 4, category ratings obtained with an expanded range of categories obtained from the numerical range of magnitude estimates (whole numbers 2-50) are plotted as a function of the same magnitude estimates. These category ratings are linearly related to the magnitude estimates, in marked contrast with the curved function in Fig. 3 above. The Peters index of curvature for this function is -0.007 , well below his criterion for curvature and close to the value zero, which would indicate a straight line.

The relationship shown in Fig. 4 has been corroborated by the results of an additional similar experiment performed with an additional group of 11 Os. The function appears essentially identical when using the data from either group. Moreover, of the total group of 22, there were 12 Os whose magnitude estimates actually fell between the limits 2 and 50 . When the means of their category ratings (Categories 2 and 50) are plotted against their magnitude estimates, a straight line with a slope of 1.0 results, having no measurable curvature and having scatter that is considerably reduced over that in Fig. 4.

\section{DISCUSSION}

Stimulus properties, such ss spacing intencities exceeding the two standards in order to eliminate end effects.

\section{RESULTS}

Figure 2 show marnitude estimates of electric tactile stimuli plotted as a function of peak current. The data points are geometric means acrose 11 Os. As a firt approximation, apparent magnitude grow a power function of stimulus intensity. The slight downward curvature at low timulus intencitie would be eliminated by correcting for threahold (Gibson, Dillman, Tomko, 1968). The value of the exponent, 2.1 , is somewhat leas than the usual 3.5 reported for electric stimuli (Gibson, Dillman \& Tomko, 1968; Hawkes, 1960; Stevens, S. S., Carton, Dhickman, 1958). The gentle slope is not unumual in light of the wide ran of intenvitie which was prewented for judiment (cf. Poulton, 1868).

Figure 3 how category rating obtained with the restricted range of categories (whole number $2-8$ ), plotted a a function of magnitude entimates of the same stimuli. The data points are arithmetic means of the category rating. The function is concave downward. The Peters (1946) index of curvature 1 for this function is -0.178 , well above the -0.10 minimum criterion value which Peters established as indicating a substantial amount of downward curvature.

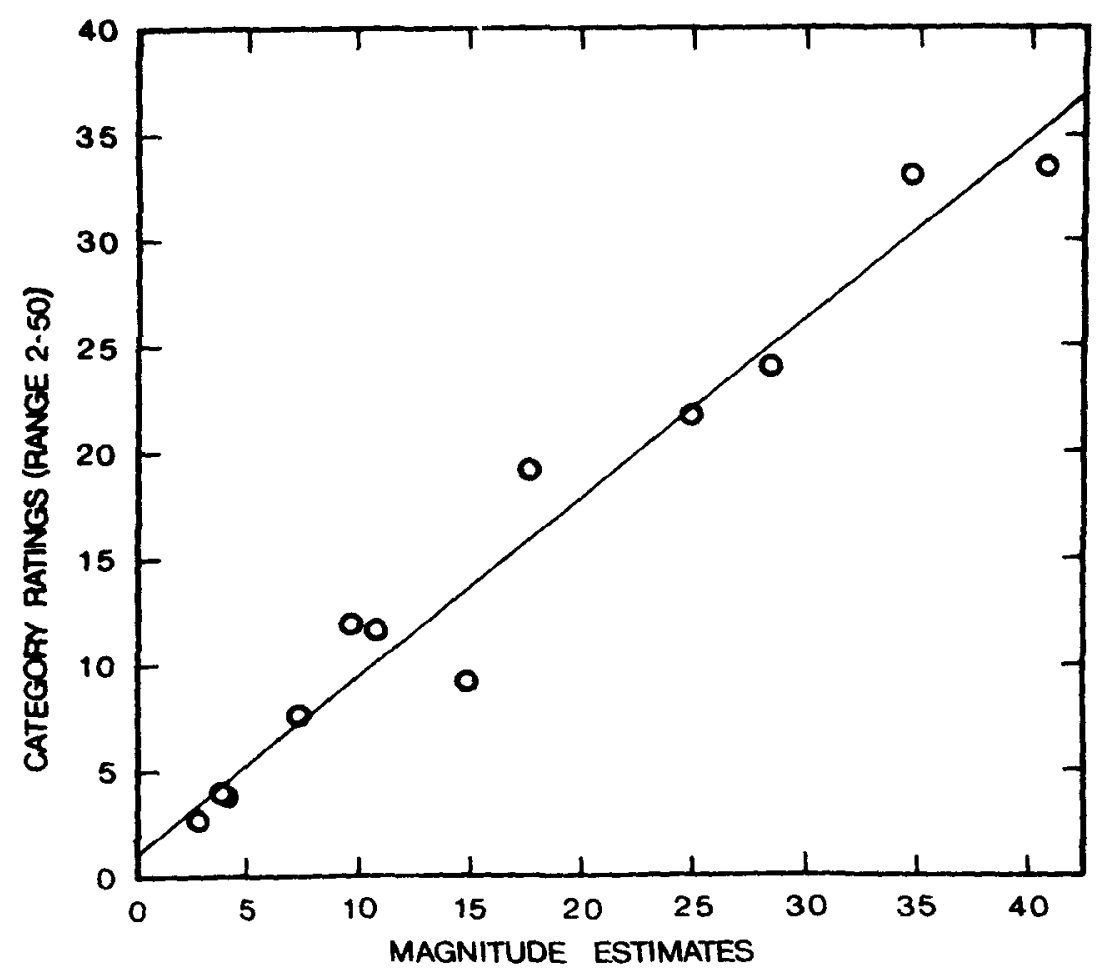

Ftg. 4. Arithmetic mean caterory ratings plotted as a function of geometric mean magnitude etimates of electrotactile stimuli between 2 and $13 \mathrm{~dB}$ above threahold. Categories were the whole numbers $2 \cdot 50$. 
(cf. Poulton, 1968; Stevens, J. C., 1958), have been found to influence the nature of the magnitude estimation function. However, the parallel effects of response dimension properties are not yet fully understood. Yet, the curvature of category scales plotted against magnitude estimates of the same stimuli has been held to distinguish prothetic from metathetic continua. It is obvious that response constraints implicit in direct estimation procedures are not the same as those operating in category scaling methods. The effects of such constraints on the form of the category scale, and thus on the nature of the relation between magnitude and category scales, are not obvious.

Direct estimation procedures permit relatively free numerical expression of sensory magnitudes from a theoretically infinite and continuous range of numbers. ${ }^{2}$ By contrast, in the typical category scaling experiment, the range and number of categories available to $O$ is set by $E$, and thus is limited and arbitrary. The integers 2-7 are frequently used as categories; both the range and the spacing between successive numbers are fixed. Given two methods of estimating sensory magnitudes and assuming a common underlying intensive continuum, it is reasonable to expect a linear function to relate them if the response constraints are effectively similar.

In the present experiment, when the limited arbitrary set of response categories was replaced by an expanded set of O-determined categories, the curved relation between category and magnitude functions became linear. This finding increases the generality of some form of the power function as being an appropriate description of the growth of apparent intensity. The result holds only within the limits of the response range used, of course; this means that no information is available about the absolute maximum range within which the linear relation holds (although there should be nothing magic about the range 2-50). The generality to other sensory scales, and answers to questions such as the relative extent to which the linearity results from an expansion of the number of categories (rather than, say, simply an increase in the numerical response range) remains to be experimentally determined.

The major point of this paper is the suggestion that the previous curved relation found between magnitude and category scales is an artifact of the restricted range of response categories supplied to the $O$. While it might be argued that setting the category ranges to be the same for both methods fixed the slope at 1.0 , the relevant point is not the value of the slope but the fact that every intermediate response was approximately the same under both scaling methods, thus removing the curvature normally expected. The suggestion has previously been made that "the nonlinearity of category scales when plotted on the same coordinates as the power functions is related to the report system [Gibson, 1960 , p. 12]." Marks suggested, "When adjusted by means of an additive constant, category scales can be described as power functions of stimulus intensity [Marks, 1968, p. 479]." From the present results, we might expect that such a corrective additive constant, as a measure of disparity between response constraints in the two scaling operations, reduces to zero when the set of categories assigned matches the set of numbers $O$ reports as magnitude estimates. The curvilinear relation between category and magnitude scales should be seen, not as a criterion for prothetic continua, but as reflecting the noncorrespondence of the range and/or grain of the respective response dimensions.

However, we are still left with the problem of the mathematical meaning of the scale units. In the present case, where one set of categories was arranged to be set equal to another set of responses, the resulting linear function implies exact correspondence between the numbers given to magnitudes on both scales. Yet, one set of responses was obtained by instructing $O$ to give equal ratios, the other by instructions aimed at obtaining equal differences between adjacent scale steps. We are reminded of Torgerson's (1958) understated caution, after he had scaled lightness and darkness both by magnitude and by category methods, that the E's instructions to form ratios may not be sufficient to conclude that the reports bear ratio relations among themselves.

\section{REFERENCES}

GIBSON, R. H. Frequency of tactile vibration and direct estimation of sensory magnitude. Unpublished MA thesis, University of Virginia, 1960.

GIBSON, R. H. Electrical stimulation of pain and touch. In D. R. Kenshalo (Ed.), The skin senses. Springfield, Ill: Thomas, 1968. Pp. 223-261.

GIBSON, $\mathbf{R}$, $H$ DILIMANN, C. $R$., TOMKO, D. L. Magnitude estimation of pulse electrical touch stimulation as a function of intensity and duration. Paper presented at the meeting of the Psychonomic Society, Chicago, Illinois. October 1967.

HAWKES, G. R. Cutaneous communication: Absolute identification of electrical intensity level. Joumal of Psychology, 1960, 49, 203-212.

MARKS, $L$. E. Stimulus-range, number of categories. and form of the category-scale. American Journal of Paychology, 1968, 81, 467-479.

PETRRS, C. C. A new descriptive statistic: The parabolic correlation coefficient. Psychometrika, 1946, 11, 67-69.

POULTON, E. C. The new psychophysics: Six models for magnitude estimation Psychological Bulletin, 1968, 69, 1-19.

STEVENS, J. C. Stimulus spacing and the judgment of loudness. Journal of Experimental Psycholory, 1958, 56, 246-250.

STEVENS, J. C., \& HALL, J. W. Brightness and loudness as functions of stimulus duration. Perception \& Psychophysics, 1966, 1, 319-327.

STEVENS, S. S., GALANTER, E. Ratio scales and category scales for a dozen perceptual continua. Journal of Experimental Psycholosy, 1957, 54, 377-411.

STEVENS, S. S., CARTON, A. S., \& SHICKMAN, G. M. A scale of apparent intendity of electric shock. Journal of Experimental Paycholosy, 1968, 56, 328-334.

TORGERSON, W. S. Theory and methode of scaling. New York: Wiley, 1858.

\section{NOTES}

1. We are indebted to $W$. $S$. Ray for advice on this procedure. The Peters index of parabolic curvature was chosen to indicate emplrically (and independently of scale values) the amount of downward curvature. The index is not influenced by the physical expansion of the ordinate scale in Fig. 2.

2. However, in practice, the presumably infinite series of positive fractions available to $O$ turns out to be quite finite. We have observed both in the present experiment and in others that $O s$ tend to use whole numbers.

(Accepted for publication April 8, 1972.) 